\title{
Medical Incidents and Evacuations on Wilderness Expeditions for the Northwest Outward Bound School
}

\author{
Forrest C. Wells, BS; Craig R. Warden, MD, MPH, MS \\ From Oregon Health \& Science University, Portland, OR (Mr Wells); and the Department of Emergency Medicine, Oregon Health \& Science \\ University, Portland, OR (Dr Warden).
}

\begin{abstract}
Introduction-Outdoor education (OE) expeditions travel far from definitive care and have unique epidemiology. Most OE expedition studies have examined a single organization and results may not generalize. This study examines the injuries, illnesses, medical evacuations, and nonmedical incidents of the Northwest Outward Bound School (NWOBS) to broaden our understanding and demonstrate commonalities within the field.

Methods - This retrospective database review examined incidents and evacuations on NWOBS expeditions from June 1, 2014 through October 31, 2016. Incident rates, evacuation rates, and incident type frequencies were calculated. Frequencies of incidents during different expedition time periods were compared with a 1 -sample $\chi^{2}$ test. The odds ratio that each type of incident would require evacuation was calculated and compared with other incident types using Fisher exact test.

Results-The study period included 59,058 program days, 277 incidents, 143 medical incidents, 75 medical evacuations, and no fatalities. Injuries occurred at a rate of 1.64 per 1000 program days and illnesses at a rate of 0.78 per 1000 program days. The most common injuries were strains, sprains, and trauma or infection of the skin and soft tissue. Most injuries occurred while backpacking, hiking, or moving around camp. The most common illnesses were nausea, vomiting, diarrhea, abdominal pain, asthma, respiratory infections, and urinary tract infections. The medical incidents with the highest odds of evacuation were fractures, urinary tract infections, abdominal pain, and asthma.

Conclusions-Results from the NWOBS database are consistent with those from other expeditionary OE programs. These findings should guide risk-management strategies and staff medical training.
\end{abstract}

Keywords: wilderness medicine, epidemiology, first aid, injury, illness, evacuation

\section{Introduction}

Expeditionary outdoor education (OE) is a wilderness activity that utilizes multiday backcountry courses to teach leadership, environmental science, and technical outdoor skills. These courses represent unique management challenges because they are typically longer, more demanding, and further from definitive care than most outdoor recreation. Moreover, participant demographics, field staff experience, and organizational risk-

Poster presentation at the WMS Meeting \& Wilderness Medicine Conference, Midway, UT, August 6, 2018.

Corresponding author: Forrest Wells, 3336 Southeast 15th Ave., Portland, OR 97202; e-mail: forrestcwells@ gmail.com.

Submitted for publication January 2018.

Accepted for publication July 2018. management strategies may change patterns of medical incidents compared with independent outdoor recreation.

Few studies have examined the epidemiology of $\mathrm{OE}$ expeditions. Most of the research has utilized the National Outdoor Leadership School (NOLS) incident database, ${ }^{1-4}$ and it is unclear whether these results can be generalized to other expeditionary OE programs. Other studies have examined a college outdoor program, ${ }^{5}$ an international trekking program, ${ }^{6}$ and Outward Bound in the 1980s ${ }^{7}$; however, these programs had meaningfully different expedition activities and organizational risk-management strategies compared with most current OE expeditions, and further research is warranted to determine if conclusions drawn from prior publications can be applied to other OE expeditions.

The Northwest Outward Bound School (NWOBS) is an expeditionary OE program based in Portland, OR that 
has offered courses in Oregon, Washington, and Idaho since 1965 with the mission of changing lives through challenge and discovery. NWOBS course activities include backpacking, mountaineering, rock climbing, whitewater rafting, sea kayaking, sailing, and canoeing. Course lengths range from 5 to $65 \mathrm{~d}$; most courses last 15 to $30 \mathrm{~d}$ and serve 8 to 10 students. Most courses are for students aged 14 to $18 \mathrm{y}$, but NWOBS also offers courses for adults and for military veterans. All field staff are wilderness first responders (WFRs). WFR is an unregulated certification offered by independent companies that typically represents a 70 - to 80 -h course in wilderness medicine and must be renewed with a 16-h refresher course every 2 y Given the remote nature of expeditions, field staff are responsible for evaluating and managing minor medical complaints, recognizing serious illness or injury, and using cell or satellite phones to seek medical direction or evacuation assistance when necessary.

NWOBS collects detailed information about incidents that occur on course for internal quality improvement and implemented a new incident database in June 2014 to facilitate data analysis. This study examines all incidents that occurred on NWOBS courses from June 1, 2014 through October 31, 2016. The results can inform strategies for risk management and medical training for similar programs and provide insight about OE expeditions to the wilderness medicine community and to individuals considering participation in these expeditions.

\section{Methods}

The NWOBS incident database includes all events since June 1, 2014 that meet any of the following criteria: require medical care beyond simple first aid, will likely require follow-up care, result in a participant being unable to participate in course activities for $\geq 1 \mathrm{~d}$, require an evacuation, involve a "near miss" wherein serious injuries were narrowly avoided, significantly affect the experience of a participant owing to behavioral or motivational problems from another participant, involve a vehicular collision, or result in deviation from NWOBS safety policy. When a reportable incident occurs, field staff record the details on a paper form immediately and review reports with management personnel during a course-end debrief. Management personnel then enter the form into an online incident database. Each report includes both categorical data classifying the incident and a narrative description written by field staff.

For this study, data were exported from the database as a comma-separated value file, imported into Excel 2016 (Microsoft Corp, Redmond, WA) and deidentified under supervision of NWOBS management. One author
(FW) read narrative descriptions of each incident to ensure that categorical data were classified correctly. The classification scheme is based on the NWOBS database methodology and modified when possible to align with terminology used in the NOLS database for consistency across studies. Incidents are classified first by category: injuries involve bodily harm from an external force and also skin and wound infections, illnesses involve disruption of normal physiological functioning, near misses involve narrowly avoiding serious injuries, behavioral incidents involve unsafe or inappropriate actions, motivational incidents stem from lack of desire to participate in course activities, vehicular incidents are reported for all collisions regardless of severity, and other incidents involve deviation from NWOBS policy or reportable circumstances that do not fall into another category. Each incident is reported in only 1 category; if multiple factors contributed, precedence is given in the order the categories are listed previously. Medical incidents with more than 1 participant or etiology are entered as separate incidents. Medical incidents with more than 1 manifestation of a single etiology are entered as 1 incident with multiple types.

Injuries are classified further by type, involved body part, and activity at the time of injury. "Burns" include sunburns and thermal injury. "Insect bites and stings" are categorized as injuries if there was only a local reaction and as the illness "anaphylaxis" if there was a severe, systemic reaction. "Skin/Wound infections" are listed as injuries for consistency with previously published database studies; other infections are listed as illnesses.

Medical evacuations occur when a participant must leave the field for assessment, treatment, or because they cannot participate in course activities. They are frequently ambulatory but may require emergent transportation. Many participants return to the expedition after receiving medical attention. Evacuations are good measures of incident severity because they indicate incidents that cannot be managed in the field and are meaningful outcomes that disrupt the expedition.

This study's data do not include identifying information and it was granted an exemption by the Oregon Health \& Science University institutional review board. Data were analyzed with SPSS 24.0 (IBM Corp, Armonk, NY). Incident rates were calculated per 1000 person-days of exposure, or "program days." Central tendency of participant age was described using the median owing to nonnormal distribution of the data. To determine if incidents occurred earlier or later in the course, the percent of course completed at the time of each incident was calculated, incidents were stratified into 5 groups by percentage of course completed, and groups were compared with a 
1-sample $\chi^{2}$ test. To assess how incident type related to evacuations, the odds ratio that each type would require evacuation was calculated and Fisher exact test was used to determine if each type had significantly different proportions of evacuations compared with the others. All tests were 2-tailed with significance set at 0.05 .

\section{Results}

The study period included 59,058 program days, 277 total incidents, 143 medical incidents, and no fatalities. Table 1 shows incident and evacuation rates. Table 2 shows the frequency of each incident category. All vehicular incidents involved low-velocity collisions resulting in minor vehicular damage and no injuries. Students had 122 medical incidents (85\%) and staff had 21 $(15 \%)$. Male participants accounted for 74 medical incidents (52\%) and female participants for 69 (48\%). Participants with medical incidents had median age of $17 \mathrm{y}$ (range 12-72) years. Medical incidents were not related to the percent of course completed $\left(\chi^{2}[4]=6.90\right.$; $P=0.14)$. Figure 1 shows the distribution of course completion at time of medical incident.

\section{INJURIES}

Table 3 shows the frequency of each injury type, Table 4 shows the anatomic locations of injuries, and Table 5 shows the activities at time of injury. "Course end run" refers to an 8- to $16 \mathrm{~km}$ trail run without packs on the last day of the expedition and "in solo camp" refers to a 24- to 48-h period where participants camp alone with intermittent supervision from field staff; both occur on

Table 1. NWOBS incident and evacuation rates, 2014-2016

\begin{tabular}{|c|c|c|c|}
\hline & \multicolumn{3}{|c|}{ Program days } \\
\hline & \multicolumn{3}{|c|}{ Incidents (rate per 1000 program days) } \\
\hline Injuries & $79(1.71)$ & $18(1.39)$ & $97(1.64)$ \\
\hline Injury evac & $36(0.78)$ & $7(0.54)$ & $43(0.73)$ \\
\hline Illnesses & $43(0.93)$ & $3(0.23)$ & $46(0.78)$ \\
\hline Illness evac & $30(0.65)$ & $2(0.15)$ & $32(0.54)$ \\
\hline $\begin{array}{l}\text { Nonmedical } \\
\text { incidents }^{a}\end{array}$ & $107(2.32)$ & $27(2.09)$ & $134(2.27)$ \\
\hline Nonmedical evac & $30(0.65)$ & $0(0)$ & $30(0.51)$ \\
\hline Medical incident totals & $122(2.64)$ & $21(1.63)$ & $143(2.42)$ \\
\hline Medical evac totals & $66(1.43)$ & $9(0.70)$ & $75(1.27)$ \\
\hline
\end{tabular}

${ }^{a}$ Near miss, behavioral, motivational, and other incidents.

NWOBS, Northwest Outward Bound School; evac, evacuations. most NWOBS courses. Injuries most commonly involved muscles, ligaments, or tendons $(46 \%)$, or skin and soft tissues (37\%). Injuries involving other structures accounted for only $17 \%$ of the total.

Table 2. Frequency of incident categories

\begin{tabular}{lcc}
\hline Incident category & Number & Percentage \\
\hline Injury & 97 & 35.0 \\
Near miss & 54 & 19.5 \\
Illness & 46 & 16.6 \\
Behavioral & 43 & 15.5 \\
Motivational & 17 & 6.1 \\
Vehicular & 6 & 2.2 \\
Other & 14 & 5.1 \\
\hline Total & 277 & 100.0 \\
\hline
\end{tabular}

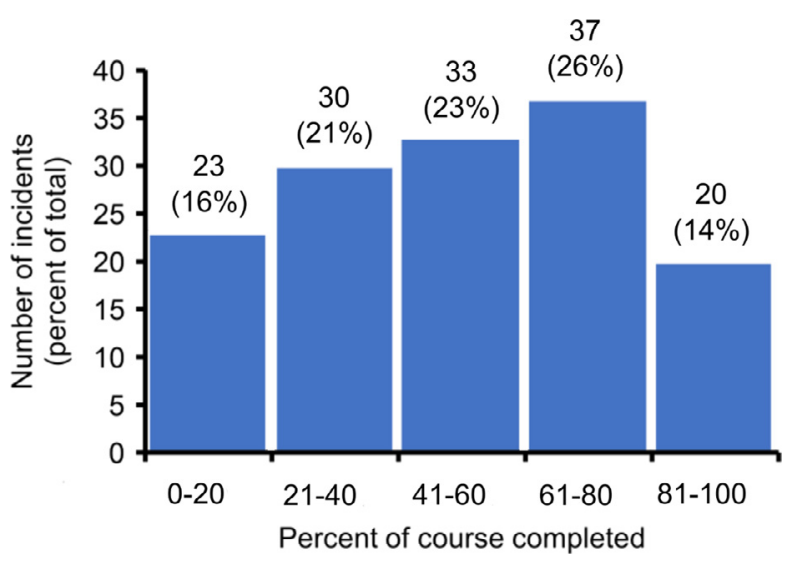

Figure 1. Percent of course completed at time of incident.

Table 3. Frequency of injury types

\begin{tabular}{|c|c|c|}
\hline Injury type & Number & Percentage \\
\hline Sprain/Strain & 45 & 46.4 \\
\hline Laceration/Puncture/Abrasion/Contusion & 18 & 18.6 \\
\hline Skin/Wound infection & 8 & 8.2 \\
\hline Blister & 4 & 4.1 \\
\hline Head injury, no loss of consciousness & 4 & 4.1 \\
\hline Burn & 3 & 3.1 \\
\hline Dislocation & 3 & 3.1 \\
\hline Fracture & 3 & 3.1 \\
\hline Insect bite or sting & 3 & 3.1 \\
\hline Dental & 2 & 2.1 \\
\hline Other $^{a}$ & 4 & 4.1 \\
\hline Total & 97 & 100.0 \\
\hline
\end{tabular}

\footnotetext{
${ }^{a} 1$ each of corneal abrasion, ruptured eardrum, testicular torsion, and nonfreezing cold injury.
} 
Table 4. Anatomic location of injuries

\begin{tabular}{|c|c|c|}
\hline Injury location & Number & Percentage \\
\hline Ankle & 20 & 20.6 \\
\hline Knee & 19 & 19.5 \\
\hline Foot & 16 & 16.5 \\
\hline Hand & 8 & 8.2 \\
\hline Back & 5 & 5.2 \\
\hline Head & 5 & 5.2 \\
\hline Wrist & 4 & 4.1 \\
\hline Arm & 3 & 3.1 \\
\hline Face & 3 & 3.1 \\
\hline Leg & 3 & 3.1 \\
\hline Mouth & 2 & 2.1 \\
\hline Shoulder & 2 & 2.1 \\
\hline Thigh & 2 & 2.1 \\
\hline Chest & 2 & 2.1 \\
\hline Other $^{a}$ & 3 & 3.1 \\
\hline Total & 97 & 100.0 \\
\hline
\end{tabular}

${ }^{a} 1$ each of eye, testicle, and no location listed.

Table 5. Activity at time of injury

\begin{tabular}{|c|c|c|c|}
\hline \multirow[b]{2}{*}{ Activity } & \multicolumn{3}{|c|}{ Incidents (percent of total) } \\
\hline & All participants & Students & Staff \\
\hline Backpacking & $33(34.0)$ & $32(40.5)$ & $1(5.6)$ \\
\hline In camp & $16(16.5)$ & $11(13.9)$ & $5(27.8)$ \\
\hline Rafting & $11(11.3)$ & $7(8.9)$ & $4(22.2)$ \\
\hline Sea kayaking & $8(8.5)$ & $6(7.6)$ & $2(11.1)$ \\
\hline Hiking without pack & $5(5.2)$ & $3(3.8)$ & $2(11.1)$ \\
\hline Mountaineering & $5(5.2)$ & $4(5.1)$ & $1(5.6)$ \\
\hline Cooking & $4(4.1)$ & $4(5.1)$ & $0(0)$ \\
\hline In solo camp & $4(4.1)$ & $4(5.1)$ & $0(0)$ \\
\hline Course end run & $3(3.1)$ & $2(2.5)$ & $1(5.6)$ \\
\hline Rock climbing & $2(2.1)$ & $2(2.5)$ & $0(0)$ \\
\hline Sailing & $2(2.1)$ & $1(1.3)$ & $1(5.6)$ \\
\hline Other $^{a}$ & $4(4.1)$ & $3(3.8)$ & $1(5.6)$ \\
\hline Total & $97(100.0)$ & $79(100.0)$ & $18(100.0)$ \\
\hline
\end{tabular}

${ }^{a} 1$ each of canoeing, on NWOBS property, expedition preparation, and ropes course.

NWOBS, Northwest Outward Bound School.

All dislocations occurred in participants with a prior history of dislocations and were reduced in the field.

Over half of the injuries were located on the knee, ankle, or foot. Fracture locations included finger, wrist, and ankle. Most of the injuries occurred while backpacking, hiking without a pack, or spending time in camp; activities traditionally considered more high risk, such as rock climbing, mountaineering, and whitewater rafting accounted for fewer than $25 \%$ of injuries.
Table 6. Frequency of illness types

\begin{tabular}{lcc}
\hline Illness type & Number & Percentage \\
\hline Nausea/Vomiting/Diarrhea & 11 & 19.3 \\
Abdominal pain & 7 & 12.3 \\
Asthma & 6 & 10.5 \\
Urinary tract infection & 6 & 10.5 \\
Upper respiratory infection & 5 & 8.8 \\
Dehydration & 4 & 7.0 \\
Mental health & 4 & 7.0 \\
Allergy & 3 & 5.3 \\
Illness infection & 3 & 5.3 \\
Anaphylaxis & 2 & 3.5 \\
Hypothermia & 2 & 3.5 \\
Other & \\
\hline Total & 4 & 7.0 \\
\hline \multicolumn{2}{c}{${ }^{a}$ each of heat exhaustion, lower respiratory tract infection, consti- } \\
pation, and syncope.
\end{tabular}

\section{ILLNESSES}

Table 6 shows the frequency of each illness type. The sum of these frequencies is greater than the number of illness incidents because some illnesses had multiple types. The most common illnesses were nausea, vomiting, diarrhea, and abdominal pain. Most narrative reports of nausea, vomiting, and diarrhea were consistent with gastroenteritis. Asthma, urinary tract infections, and upper respiratory infections were also common. Illnesses were classified as asthma only in participants with a prior diagnosis. Illnesses were classified as urinary tract infection only when diagnosed at a health care facility after evacuation. Hypothermia and heat exhaustion were less common and no cases were severe. Mental illnesses included posttraumatic stress disorder, anxiety, and depression. Illness infections included conjunctivitis, sinusitis, pharyngitis, and lymphadenitis. The most serious illnesses were 2 cases of anaphylaxis, both due to hymenoptera venom.

\section{MEDICAL EVACUATIONS}

There were 75 medical evacuations, with a rate of 1.27 medical evacuations per 1000 program days. Medical incidents resulted in an evacuation $52 \%$ of the time. Table 7 lists medical incident types with 3 or more cases and shows the percent evacuated and odds ratio of evacuation for each type.

\section{BEHAVIORAL INCIDENTS}

Table 8 shows the frequency of each behavioral incident type. The sum of these frequencies is greater than the 
Table 7. Medical evacuations

\begin{tabular}{|c|c|c|c|c|}
\hline Incident type & Number of incidents & Percent evacuated & Odds ratio of evacuation & $P<0.05$ \\
\hline Urinary tract infection & 6 & 100 & $\mathrm{~N} / \mathrm{A}^{a}$ & $b$ \\
\hline Fracture & 3 & 100 & $\mathrm{~N} / \mathrm{A}^{a}$ & - \\
\hline Abdominal pain & 7 & 86 & 5.83 & - \\
\hline Asthma & 6 & 83 & 4.79 & - \\
\hline Upper respiratory infection & 5 & 80 & 3.77 & - \\
\hline Skin/Wound infection & 8 & 75 & 2.87 & - \\
\hline Blister & 4 & 75 & 2.79 & - \\
\hline Allergy & 3 & 67 & 1.84 & - \\
\hline Illness infection & 3 & 67 & 1.84 & - \\
\hline Nausea/Vomiting/Diarrhea & 11 & 55 & 1.10 & - \\
\hline Mental health & 4 & 50 & 0.90 & - \\
\hline Sprain/Strain & 45 & 44 & 0.63 & - \\
\hline Burn & 3 & 33 & 0.45 & - \\
\hline Dislocation & 3 & 33 & 0.45 & - \\
\hline Laceration/Puncture/Abrasion/Contusion & 18 & 33 & 0.41 & - \\
\hline Dehydration & 4 & 25 & 0.29 & - \\
\hline Insect bite or sting & 5 & 20 & 0.22 & - \\
\hline Head injury, no loss of consciousness & 4 & 0 & 0 & $b$ \\
\hline
\end{tabular}

N/A, not applicable.

${ }^{a}$ The odds ratio is undefined as calculation would require division by 0 .

${ }^{b}$ Fisher exact test indicates significantly different proportions of evacuations with $P<0.05$.

Table 8. Frequency of behavioral incident types

\begin{tabular}{lcc}
\hline Behavior type & Number & Percentage \\
\hline Aggression & 14 & 31.1 \\
Suicidal ideation/Self-harm & 12 & 26.7 \\
Inappropriate language & 5 & 11.1 \\
Running away & 5 & 11.1 \\
Disobedience & \\
Drug/Alcohol use & 4 & 8.9 \\
Theft & 3 & 6.7 \\
Total & 2 & 4.4 \\
\hline
\end{tabular}

\footnotetext{
${ }^{a}$ Typically resulting in unsafe situations from failure to follow instructions.
}

number of behavioral incidents because some behavioral incidents were assigned multiple types. Incidents involving aggression ranged from verbal threats to physical assault. Incidents involving suicidal ideation and selfharm ranged from passive suicidal ideation without plan or intent to minor self-harm behaviors. There were no suicide attempts or self-harm behaviors requiring more than basic first aid.

\section{Discussion}

Although many individuals participate in $\mathrm{OE}$ expeditions every year, there are few epidemiological studies of these courses. The NOLS incident database forms most of the current knowledge base but without publications derived from databases of similar OE programs it is unclear whether results can be generalized across the field. Organizations such as NWOBS and NOLS have experience maintaining incident report databases and using them to guide and monitor internal quality improvement projects. By making their results public they provide valuable information to other OE programs and to the wilderness medicine community. To that end, this study examined nearly $3 \mathrm{y}$ of data from the NWOBS incident database in an attempt to broaden the understanding of the epidemiology of OE expeditions.

Authors of a prior epidemiological study of $\mathrm{OE}$ expeditions cautioned against directly comparing incident data from different programs, as "differences in organizational mission, thresholds for reportable incidents, and other factors make [that] type of comparison inappropriate." 2 Although judging the safety of different programs from incident databases may be misguided, comparison of this data with the literature yields valuable insights for the wilderness medicine community. Hopefully, similarities between programs will provide evidence that can be generalized across $\mathrm{OE}$ and differences will be used to start conversations aimed at information sharing and mutual improvement. The resulting discussion should help OE programs develop safety management policies, staff 
trainings, and incident response plans that improve the health and safety of all participants on expeditionary courses.

In alignment with findings from other expeditionary OE programs, the overall rate of medical incidents on NWOBS courses is low. Indeed, there were remarkably similar rates of medical incidents per 1000 program days between NWOBS (2.42), NOLS (2.26), ${ }^{3}$ and a collegiate OE program $(1.50)^{5}$ when compared with college athletics $(5.56)^{8}$ or an international trekking program (43.91). ${ }^{6}$ This may be due to extensive risk-management strategies and careful course element selection by expeditionary OE programs or may reflect low incident rates in the outdoor activities common on OE expeditions. Examples of organizational risk-management strategies are discussed below and also include annual staff trainings in expedition activities, detailed safety policies, and mentoring of staff safety planning.

The most common types of incidents for NWOBS and NOLS are also similar. Table 9 shows the 3 most common injuries, illnesses, activities at time of incident, and nonmedical incidents in this study and in 2 recent NOLS database studies. Although the NOLS database studies use different names for several incident types, review of their methodology indicates that the defining criteria are the same across studies for each of the incident types listed in Table 9. Key comparisons are discussed by subsection below.

\section{TIMING OF INCIDENTS}

Prior research found medical incidents to be more frequent during the first $10 \mathrm{~d}$ of expeditions; sprains and strains were particularly common during this period, likely because of poor physical conditioning. ${ }^{2}$ Many of the courses in the NWOBS database were shorter than $4 \mathrm{wk}$ so more students were exposed to course days 1 to 10 than to course days 20 to 30 , and the percentage of incidents occurring during the first $10 \mathrm{~d}$ was not a meaningful value in this study. Instead, testing for an association between percentage of course completed and incident frequency found that incidents were not more frequent at the beginning of course. This might be because most NWOBS courses begin with a week of

Table 9. Comparison of incident types

\begin{tabular}{|c|c|c|c|}
\hline \multirow[b]{2}{*}{ Category } & \multicolumn{3}{|c|}{ Percent of total within category (rank order of frequency within category) } \\
\hline & NWOBS 2014-2016 & NOLS 2002-2005 & NOLS 1999-2002 2 \\
\hline \multicolumn{4}{|l|}{ Injuries } \\
\hline Sprain/Strain & $46.4(1)$ & $55.2(1)$ & $49.9(1)$ \\
\hline Skin/Soft tissue (all) ${ }^{a}$ & $37.1(2)$ & $27.4(2)$ & $30.8(2)$ \\
\hline Head injury, no loss of consciousness & $4.1(3)$ & $1.7(6)$ & $3.8(3)$ \\
\hline Dental & $2.1(6)$ & $5.2(3)$ & $3.2(6)$ \\
\hline \multicolumn{4}{|l|}{ Illnesses } \\
\hline Nausea/Vomiting/Diarrhea & $19.3(1)$ & $23.6(1)$ & $26.4(1)$ \\
\hline Abdominal pain & $12.3(2)$ & $6.9(5)$ & $6.8(5)$ \\
\hline Asthma & $10.5(3)$ & $2.1(10)$ & $1.9(10)$ \\
\hline Urinary tract infection & $10.5(3)$ & $5.9(7)$ & $6.2(7)$ \\
\hline Respiratory infection $^{b}$ & $10.5(3)$ & $14.6(3)$ & $16.6(2)$ \\
\hline Illness infection & $5.3(8)$ & $15.7(2)$ & $11.1(3)$ \\
\hline \multicolumn{4}{|l|}{ Activity at time of incident } \\
\hline Backpacking & $34.0(1)$ & $43.8(1)$ & $46.1(1)$ \\
\hline In camp & $16.5(2)$ & $12.2(2)$ & $17.1(2)$ \\
\hline River boating $^{c}$ & $11.3(3)$ & $3.9(5)$ & $4.1(3)$ \\
\hline Rock climbing & $2.1(10)$ & $5.0(3)$ & $2.0(10)$ \\
\hline \multicolumn{4}{|l|}{ Incident categories } \\
\hline Medical & $51.6(1)$ & Not reported & $63.7(1)$ \\
\hline Near miss & $19.5(2)$ & Not reported & $21.7(2)$ \\
\hline Behavioral & $15.5(3)$ & Not reported & $3.1(4)$ \\
\hline Motivational & $6.1(4)$ & Not reported & $8.0(3)$ \\
\hline
\end{tabular}

\footnotetext{
${ }^{a}$ Includes laceration/puncture/abrasion/contusion, skin/wound infection, blister, burn, and insect bite or sting.

${ }^{b}$ Includes upper respiratory infection, lower respiratory tract infection, and flu-like illness.

${ }^{c}$ Includes river rafting, canoeing, and kayaking.

NOLS, National Outdoor Leadership School; NWOBS, Northwest Outward Bound School.
} 
whitewater rafting; staff use this relatively nonphysically demanding activity and morning calisthenics to prepare students for the more challenging activities of backpacking and mountaineering that come later in the course. Alternatively, the shorter courses may not allow enough time for participants to improve their conditioning and decrease their risk of incidents later in the course, or the database may contain too few incidents for this analysis.

\section{INJURIES}

As in prior studies, ${ }^{4,5,7}$ sprains and strains were the most common injury type. Although they occurred in many situations, the most common scenario was a sprain or strain of the lower extremity while backpacking, hiking, or moving around camp. NWOBS works to prevent these injuries by decreasing pack weight, providing students with trekking poles, and discouraging risky behavior such as running with packs. Several sprains and strains were related to upper extremity overuse during paddling activities such as whitewater rafting, sea kayaking, and canoeing. These injuries are more difficult to address; strategies have focused on warming up, stretching, and proper paddling technique. Conservative expedition planning with careful progression of difficulty may help prevent sprains and strains of both upper and lower extremities. Choosing appropriate objectives has traditionally focused on technical endeavors such as summiting peaks or rafting difficult whitewater. These data suggest that staff must remember to set realistic objectives in nontechnical terrain as well.

Although fewer than half of all sprains and strains resulted in evacuations, they still accounted for more evacuations than any other incident type. Sprains and strains were rarely severe enough to require urgent medical attention, but they often prevented participants from continuing on these physically demanding expeditions. Low motivation may have also contributed to some of these evacuations, as some students likely used minor sprains and strains as justification for leaving a course.

Skin and soft tissue injuries and infections were also common, and infections had high odds of evacuation owing to the need for antibiotic therapy. Given the frequency and impact of wound infections, future work should synthesize key elements from wilderness wound care practice guidelines to be standardized across WFR courses and incorporated into staff trainings. ${ }^{9}$

Although none of the 4 head injuries involved loss of consciousness, 3 presented with symptoms concerning concussion, including headache, dizziness, difficulty with concentration and memory, and blurry vision. Staff must be able to quickly differentiate between symptoms of concussion and intracranial hemorrhage and should be knowledgeable about current guidelines for concussion management. Although protocol mandates evacuation for loss of consciousness or severe neurological deficits, management of more minor head injuries is typically left to staff discretion and may represent an area for further training.

\section{ILLNESSES}

As in other programs, ${ }^{2,3}$ the most frequent illness on NWOBS courses was nausea, vomiting, and diarrhea, typically due to gastroenteritis. Accordingly, NWOBS has implemented strategies to improve hand hygiene, cookware sanitation, and water purification. Gastroenteritis may have also caused cases of abdominal pain, but diagnoses from health care providers after evacuation indicate that 2 cases of abdominal pain were due to appendicitis and 1 to cholecystitis. Field staff with minimal medical training and no laboratory or imaging access often evacuate patients with abdominal pain for further evaluation, especially if the pain is severe, lasts longer than several days, or is accompanied by other worrisome symptoms. The health care provider's diagnosis is sometimes included in the narrative section of the incident report, but all cases are categorized as "abdominal pain" regardless of the diagnosis. None of the narrative descriptions indicated pregnancy as a cause of abdominal pain, and staff do not carry pregnancy tests in the field.

Urinary tract infections also had high odds of evacuation because of the need for antibiotic therapy. Considering that skin, wound, and urinary tract infections commonly resulted in evacuations to receive antibiotics from a health care provider, NWOBS might benefit from equipping field staff with empiric antibiotics for these conditions to administer in the field after cellular or satellite phone consultation with a medical director. Alternatively, NWOBS might recommend that participants with known susceptibility to urinary tract infections bring appropriate antibiotics on the expedition to use if necessary.

There were no life-threatening asthma attacks. However, the frequency of asthma incidents, potential for consequence, and tendency for students to forget rescue inhalers when leaving camp is concerning. Expeditionary $\mathrm{OE}$ programs would benefit from policies requiring participants to bring an extra inhaler and asthma action plan to leave with staff and protocols for the use of epinephrine autoinjectors in case of status asthmaticus. The 2 cases of anaphylaxis are sobering reminders of why staff should have ready access to epinephrine, which NWOBS provides as 2 autoinjectors per expedition.

There were relatively few instances of dehydration, heat illness, or hypothermia. NWOBS course environments 
range from mountains to deserts, and expeditions often encounter snow, freezing rain, or temperatures above $38^{\circ} \mathrm{C}$. The low rates of environmental illness may be due to proactive staff interventions, precourse advising to help students select appropriate gear, and a collection of outdoor clothing that NWOBS maintains to loan students who come to course inadequately equipped. There are few places in the NWOBS course area high enough to cause altitude illness, and travel to these locations during courses is rare.

\section{BEHAVIORAL INCIDENTS}

Behavioral incidents are common, disruptive, and emotionally harmful to both students and staff. Violence, suicidal ideation, and self-harm hold the potential for physical injury as well. Behavioral incidents likely go underreported, as student behavioral problems resulting in injury or illness are typically categorized as medical incidents. NWOBS has attempted to reduce the impact of behavioral incidents with precourse student screening, policies for managing behavioral incidents, and a multiday student management skills training for field staff annually.

The frequency of aggression, suicidal ideation, and self-harm highlight the importance of staff trainings targeted at these behaviors. Risk assessment of threat to self or others is particularly important to develop strategies for supervision, intervention, and evacuation. Suicidality and aggression may occur in other wilderness contexts as well, so elements of risk assessment should be incorporated into WFR courses.

\section{IMPLICATIONS FOR STAFF TRAINING}

As discussed above, these results align with findings from other expeditionary $\mathrm{OE}$ programs in demonstrating that medical incidents on OE expeditions are infrequent and usually one of a few common incident types. In contrast, guidelines for WFR course topics ${ }^{10}$ and a position statement regarding the WFR scope of practice ${ }^{11}$ include extensive lists of medical problems that WFRs are expected to understand and manage. NOLS, NWOBS, and many college outdoor programs require WFR certification for field staff, but current understanding of expedition epidemiology and clinical skills retention calls into question the necessity and feasibility of field staff performing the full WFR scope of practice. $^{12}$ A study of graduates of an introductory 16-hour wilderness first aid course found that clinical skills and knowledge decreased during the first year after the course, especially in novel medical topics. ${ }^{13}$ Self-perception of medical ability was not predictive of performance, raising concern that laypersons exposed to a broad scope of practice that they do not typically encounter might attempt invasive interventions or field management of complex conditions.
Further discussion of the WFR scope of practice and staff medical training requirements should emphasize providing medical care for common conditions, understanding organizational treatment protocols, and knowing when and how to call for medical direction. The financial burden of trainings on field staff should also be considered: $\mathrm{OE}$ is poorly compensated and the cost of maintaining certifications is often a substantial portion of earnings. If field staff are required to dedicate significant resources to medical training, they may be less likely to pursue additional trainings in other expedition activities. Expeditionary OE programs may benefit from frequent, basic, and inexpensive staff medical trainings.

\section{LIMITATIONS}

This study has several limitations. First, there is a potential reporting bias arising from staff decisions of which incidents meet reporting criteria. Incidents barely meeting criteria are likely underreported, and many conditions of interest such as minor blisters, rashes, or sunburns were excluded from the data if they did not meet additional criteria. Second, the small number of medical incidents prevents a more robust analysis. Third, many incidents involve multifactorial motivational, behavioral, and medical contributions; the current classification scheme does not capture this complex interplay. Fourth, incident rates by sex or by activity at time of injury could not be calculated because person-days of exposure by sex or by activity were not known. Fifth, the data are from a single organization with organizational risk-management strategies, which may limit generalizability to independent outdoor recreation.

\section{Conclusions}

Despite the longstanding popularity of OE expeditions, few studies have examined the epidemiology of these courses. Most of the research utilizes data from a single organization, raising questions of generalizability. This study broadens the scope of the research by examining data from a different program. Overall, the findings were similar between the 2 organizations, suggesting several conclusions that may generalize across expeditionary OE programs. First, rates of medical incidents are typically low. Second, the most common medical incidents are sprains and strains; skin and soft tissue injuries and infections; and nausea, vomiting, and diarrhea. Lifethreatening medical incidents are exceedingly rare. Third, most injuries occur during activities traditionally considered low risk, such as backpacking, hiking, and moving around camp. These findings speak to the ability of organizational risk-management strategies and staff skills in nonmedical domains to prevent medical 
incidents, and they suggest that staff typically utilize a fraction of the WFR scope of practice. Future work should focus on sharing effective risk-management strategies, examining medical skills retention in WFRs, and optimizing staff training to prevent and respond to common medical incidents.

Acknowledgments: We are grateful to the Northwest Outward Bound School for access to the incident data used in this study.

Author Contributions: Study concept and design (FW, CW); acquisition of the data $(\mathrm{FW})$; analysis of the data $(\mathrm{FW})$; drafting of the manuscript (FW); critical revision of the manuscript (FW, CW); and approval of final manuscript (FW, CW).

Financial/Material Support: None.

Disclosure: FW was employed as field staff by the Northwest Outward Bound School for 3 years before the conception of this study. While the study was ongoing, he was occasionally employed as event staff and earned less than US $\$ 500$ total during the study period. He received no financial compensation for his work on this study.

\section{Supplementary materials}

Supplementary data associated with this article can be found in the online version at https://doi.org/10.1016/j. wem.2018.07.004.

\section{References}

1. Gentile DA, Morris JA, Schimelpfenig T, Bass SM, Auerbach PS. Wilderness injuries and illnesses. Ann Emerg Med. 1992;21(7):853-61.

2. Leemon D, Schimelpfenig T. Wilderness injury, illness, and evacuation: National Outdoor Leadership School's incident profiles, 1999-2002. Wilderness Environ Med. 2003;14(3):174-82.

3. McIntosh SE, Leemon D, Visitacion J, Schimelpfenig T, Fosnocht D. Medical incidents and evacuations on wilderness expeditions. Wilderness Environ Med. 2007;18 (4):298-304.

4. Stanford KA, Phillips L, Chang Y, Leemon D, Schimelpfenig T, Harris NS. Trends in skin and soft tissue-related injuries in NOLS wilderness expeditions from 1984 to 2012. Wilderness Environ Med. 2017;28(4):307-12.

5. Gaudio FG, Greenwald PW, Holton M. Injury and illness in college outdoor education. Wilderness Environ Med. 2010;21(4):363-70.

6. Sadnicka A, Walker R, Dallimore J. Morbidity and determinants of health on youth expeditions. Wilderness Environ Med. 2004;15(3):181-7.

7. Paton BC. Health, safety and risk in Outward Bound. J Wilderness Med. 1992;3(2):128-44.

8. Hootman JM, Dick R, Agel J. Epidemiology of collegiate injuries for 15 sports: summary and recommendations for injury prevention initiatives. $J$ Athl Train. 2007;42(2):311-9.

9. Quinn RH, Wedmore I, Johnson E, Islas AA, Anglim A, Zafren K, et al. Wilderness Medical Society practice guidelines for basic wound management in the austere environment. Wilderness Environ Med. 2014;25 (3):295-310.

10. Wilderness Medical Society Curriculum Committee. Wilderness first responder: recommended minimum course topics. Wilderness Environ Med. 1999;10(1):13-9.

11. Wilderness Medicine Educators Collaborative. Wilderness first responder scope of practice. Available at: www.wfrsop.org. Accessed December 6, 2017.

12. Welch TR, Clement K, Berman D. Wilderness first aid: is there an "industry standard"? Wilderness Environ Med. 2009;20(2):113-7.

13. Schumann SA, Schimelpfenig T, Sibthorp J, Collins RH. An examination of wilderness first aid knowledge, self-efficacy, and skill retention. Wilderness Environ Med. 2012;23 (3):281-7. 